\title{
Myosite ossifiante du muscle temporal après polytraumatisme
}

\author{
Rapport de cas et \\ revue de la \\ littérature \\ Authors: \\ Braem D (MD) ${ }^{1, *}$ \\ Darche V (MD, DDS) ${ }^{1}$, \\ Legros $E(M D, D D S)$
}

Institutions:

${ }^{1}$ Service de chirurgie orale et maxillo-faciale, Centre Hospitalier Régional de Namur, Av. Albert Ier 185, 5000 Namur.

Auteur correspondant: Braem D, Service de chirurgie orale et maxillo-faciale, Centre Hospitalier Régional de Namur, Av. Albert Ier 185, 5000 Namur,

Dimitri.braem2010@hotmail.com, ORCID iD: orcid.org/0000-0003-4724-3619

Notice : les opinions exprimées dans ce manuscrit sont propres aux auteurs et il ne s'agit pas d'une opinion officielle d'une institution ou d'une source de financement. 


\section{Lettre à l'éditeur-en-chef}

\author{
Monsieur l'Editeur-en-chef,
}

Merci de bien vouloir recevoir cet article intitulé « Myosite ossifiante du muscle temporal après polytraumatisme » afin qu'il soit soumis à une évaluation libre par les pairs dans la revue Nemesis.

1) Résumer la contribution de cet article par rapport à la littérature scientifique: Il s'agit de documenter la littérature sur les rares cas de myosite ossifiante isolée du muscle temporal et de réaliser un résumé succinct de la littérature concernant ce sujet.

2) Expliquer le lien entre cet article et les travaux déjà publiés: Les derniers cas de myosite ossifiante des muscles de la face recensés dans la littérature sont peu récents. L'objectif est de donner un aperçu global de la pathologie et des dernières recommandations concernant les méthodes diagnostiques, les diagnostics différentiels et les traitements.

3) Spécifier le type d'article (par exemple: article original de recherche, revue, métaanalyse, essai clinique): Il s'agit d'un rapport de cas.

4) Décrire des contacts avec la revue Nemesis concernant le manuscrit soumis:

Nemesis est une revue récente qui a été conseillée par le Dr Legros, ancien assistant en chirurgie maxillo-faciale aux Cliniques Universitaires de Saint-Luc

5) Préciser en quoi l'article correspond aux attentes et objectifs de la revue Nemesis: Nemesis est une revue facilement accessible et prend également en compte des articles concernant des rapports de cas. L'intérêt de cet article serait de modestement contribuer à la qualité scientifique de la revue en proposant un cas extrêmement rare. 
Résumé

La myosite ossifiante traumatique (MOT) du muscle temporal est une pathologie extrêmement rare. Une limitation de l'ouverture buccale est le symptôme clé. Les examens d'imagerie sont très utiles au diagnostic, le CT-scanner de la face étant l'examen le plus performant pour la détection de la MOT. Le meilleur traitement est la résection chirurgicale complète. Les cas de myosite ossifiante traumatique dans la région de la tête et du cou sont rares. Les atteintes au niveau du muscle temporal sont encore plus rares. Nous présentons ici le cas d'une ossification isolée du muscle temporal gauche après un polytraumatisme. L'intérêt de l'observation clinique du cas présenté est donc de documenter la littérature. Le diagnostic différentiel et les signes pathognomoniques présents sur les examens d'imagerie seront également détaillés.

\section{Summary}

Myositis ossificans traumatica (MOT) of the temporal muscle is an extremely rare disease. A limitation of mouth opening is a key symptom. Imaging is very useful for diagnosis. The computed tomography scanner of the face is the most efficient for the detection of the MOT. Complete surgical resection is the best treatment. Cases of MOT in the head and neck area are rare. Cases of isolated temporal muscle involvement are even rarer. We present the case of an isolated ossification of the left temporal muscle after polytrauma. The interest of the clinical observation of the case presented is thus to document the literature. The differential diagnosis and pathognomonic signs present on the imaging examinations will also be detailed.

Keywords: Myositis ossificans, temporal muscle, calcification 


\section{Introduction}

La myosite ossifiante traumatique est une pathologie caractérisée par la formation d'un tissu osseux extra-squelettique [1]. Il existe au moins deux entités de myosite ossifiante. La myosite ossifiante progressive (maladie de Munchmeyer) est une maladie héréditaire à transmission autosomique dominante. Elle apparaît durant l'enfance et atteint plusieurs muscles. Elle est également habituellement accompagnée de manifestations systémiques telles que des anomalies squelettiques, des troubles du développement sexuel et une surdité.

La myosite ossifiante traumatique (MOT) est une forme plus localisée de myosite ossifiante [2]. Elle est induite par un traumatisme majeur ou de multiples traumatismes mineurs. Nous ne traiterons dans cet article que de la myosite ossifiante traumatique.

\section{Rapport de cas}

Un patient caucasien, âgé de 43 ans, a été hospitalisé en septembre 2015 pour un polytraumatisme dans le cadre d'un accident de la voie publique avec choc frontal. Il n'existait pas d'antécédents médicaux-chirurgicaux ni familiaux particuliers.

Le patient a séjourné pendant une longue période aux soins intensifs avec une ventilation mécanique de longue durée et finalement avec une trachéotomie. Au niveau neurologique, le patient a présenté un hématome temporal gauche avec déviation de la ligne médiane vers la droite et une hémorragie du ventricule latéral gauche ainsi qu'un hématome sous-dural fronto-pariéto-temporal gauche. Le patient a présenté une fracture comminutive de l'ulna gauche et une fracture métaphyso-épiphysaire en regard du condyle médial. On notait aussi une fracture transverse déplacée de la diaphyse proximale du fémur gauche.

L'évolution lors de l'hospitalisation a été marquée par un éveil important de coma. L'amélioration clinique a été notable. Ensuite, une longue période de rééducation a été nécessaire.

D'un point de vue maxillo-facial, le patient a présenté une fracture fronto-temporale gauche comminutive, avec embarrure millimétrique, une fracture de l'arcade zygomatique gauche, une fracture comminutive de la paroi externe de l'orbite gauche avec atteinte du muscle oculomoteur externe gauche, un fragment osseux intra-orbitaire en regard de la fracture, une fracture de la grande aile gauche de l'os sphénoïdal et un hémosinus maxillaire gauche, sphénoïdal et ethmoïdal. Il s'en est suivi une perte totale de la vision de l'œil gauche suite à une atteinte du nerf optique ainsi qu'une quadranopsie temporale droite. 
En juin 2017, le patient a constaté des douleurs au niveau de l'articulation temporomandibulaire gauche, à l'ouverture buccale et à l'alimentation. L'ouverture buccale était évaluée à cinq centimètres, avec progressivement, en fin d'ouverture une déviation de l'ouverture buccale vers la gauche. Lors de l'ouverture, le patient a signalé une douleur prétragienne gauche, signe d'une sensibilité au niveau de l'articulation temporo-mandibulaire gauche. La palpation des muscles masséters gauche, temporal gauche et ptérygoïdien gauche était sensible. Inversement, la palpation des muscles masséter droit, temporal droit et ptérygoïdien droit était sans particularité. Le test au mordu à droite était sans particularité et, à gauche, confirmait une sensibilité dans la région articulaire gauche.

Une radiographie panoramique a été réalisée. Elle a montré une parodontite modérée qui nécessitait une prise en charge au niveau des secteurs molaires supérieurs gauches et inferieurs droits. Les têtes condyliennes gauche et droite étaient correctement centrées au sein des cavités glénoïdes. On ne notait pas de séquelle de fracture sur base de la seule radiographie panoramique.

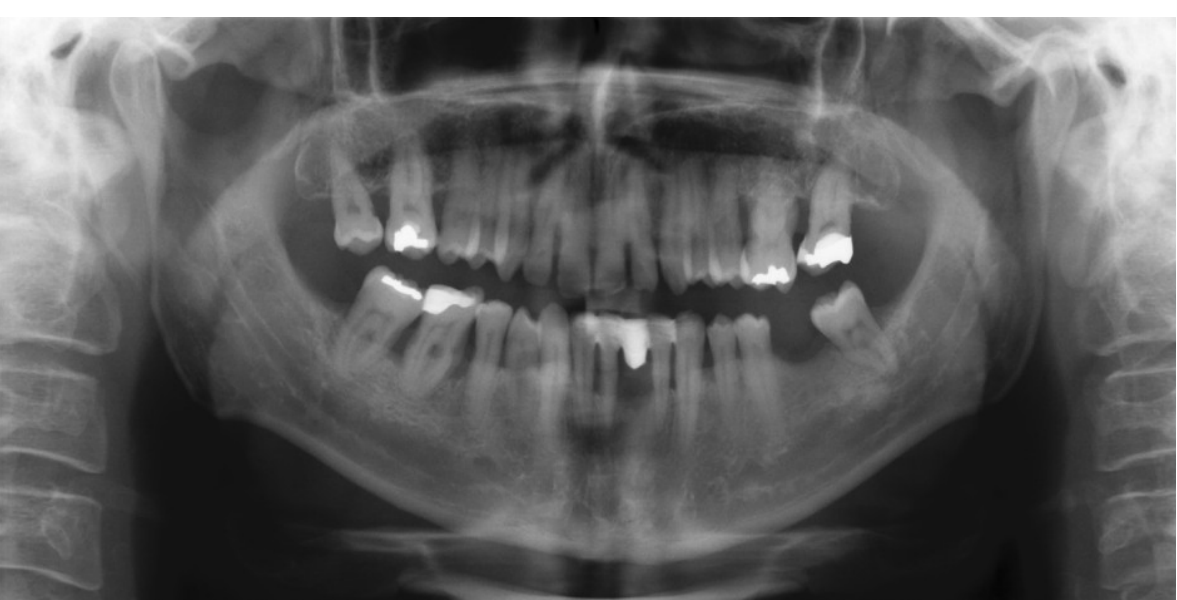

Fig. 1. Orthopantomogramme de 2017 ne montrant aucune particularité par rapport aux plaintes du patient.

Un CT scanner du massif facial a été réalisé en mars 2017, et a mis en évidence une avulsion de la dent $n^{\circ} 31$, une parodontopathie au niveau des dents $n^{\circ} 44-45-46$, une séquelle de fracture de la paroi postéro-latérale de l'orbite gauche, un remaniement arthrosique du condyle mandibulaire gauche, une suspicion de séquelle fracturaire de l'épine nasale. Des fragments osseux et esquille osseuse au sein du muscle temporal gauche ont également été remarqués. La relecture du CT scanner à l'admission et de septembre 2015 avait exclu toute fracture condylienne et tout processus dégénératif. 


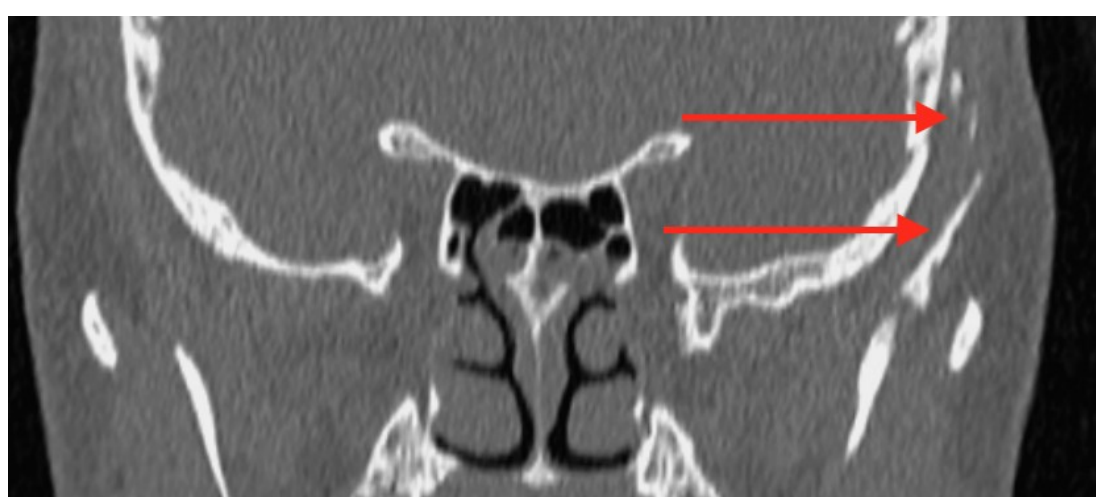

Fig. 2. CT scanner du massif facial de mars 2017 mettant en évidence des fragments osseux au sein du muscle temporal gauche (flèches).

Une RMN des articulations temporo-mandibulaires a également été demandée afin de vérifier l'intégrité discale. Cette dernière a permis d'objectiver un remaniement post-traumatique de la tête du condyle mandibulaire gauche associé à une vraisemblable luxation discale antérieure irréductible.

Une intervention chirurgicale n'est pas conseillée d'emblée, mais celle-ci n'est pas à exclure à l'avenir. La question a surtout été de savoir si dans le futur l'ossification occasionnera des limitations fonctionnelles plus importantes, et aussi des phénomènes algiques?

Un CT scanner cérébral de contrôle à 6 mois a été réalisé en octobre 2018 et a mis en évidence une ossification du muscle temporal gauche sur une hauteur d'environ $55 \mathrm{~mm}$ et avec une épaisseur de $3 \mathrm{~mm}$. Le patient a développé une myosite ossifiante du muscle temporal gauche.

\section{Discussion}

La myosite ossifiante des muscles de la face est une pathologie rare. Elle est divisée en myosite ossifiante progressive, qui est une pathologie héréditaire avec des manifestations systémiques et myosite ossifiante traumatique (MOT), qui est une pathologie plus localisée [1-13]. Nous ne discuterons ici que de la myosite ossifiante traumatique.

Elle est causée par un traumatisme majeur ou de multiples traumatismes mineurs. Plusieurs types de traumatismes ont été décrits: des extractions dentaires multiples, une migration d'un abcès odontogène, une injection d'anesthésique local, une génioplastie, un traitement orthodontique mal réalisé et un choc direct ou une 
fracture de la face [3]. L'histoire traumatique est cependant absente dans $30 \%$ des cas $[3,4]$. La MOT des muscles de la face est une localisation extrêmement rare. Le muscle le plus affecté dans cette région est le muscle masséter. Le muscle temporal est le moins affecté. Moins de 10 cas ont été recensés dans la littérature pour une atteinte isolée du muscle temporal [1].

Les causes de la MOT ne sont pas claires. La théorie la plus acceptée résulte d'une hémorragie intramusculaire avec prolifération vasculaire et formation d'un tissu de granulation qui subit une métaplasie en tissu cartilagineux ou osseux $[3,5]$.

Les symptômes surviennent généralement quelques mois après l'épisode traumatique, mais il peuvent parfois survenir plusieurs années après. Dans ce cas-ci, les symptômes apparaissent deux ans après le traumatisme initial.

Le symptôme principal est un trismus. A l'examen clinique, une asymétrie faciale, un œdème persistant, une augmentation d'une masse et de la douleur peuvent être constatées. L'absence de symptôme est aussi possible [4].

Dans le cas discuté ici, le patient ne présente pas de limitation de l'ouverture buccale mais uniquement une douleur au niveau des muscles masticateurs gauches.

Il n'existe pas de marqueur biologique spécifique de la MOT. Le diagnostic différentiel d'une lésion formée de tissus osseux comprend la fascite nodulaire, l'ostéome juxtacortical, l'ostéochondrome, l'ostéoblastome, le chondrome, le sarcome, le chondrosarcome, ou encore l'hémangiome veineux et la maladie de Jacob [4]. Il est impératif d'exclure ces lésions avant de conclure au diagnostic de MOT [6]. Le diagnostic différentiel s'obtient grâce à l'examen clinique et l'histoire traumatique antérieure, mais aussi grâce à l'aide des examens de radiodiagnostic. La radiographie panoramique peut montrer une formation de tissus osseux ectopique, mais n'est pas très efficace pour détecter sensiblement la MOT, dû à la superposition des os du crâne [7].

Le CT scanner est sensible pour détecter les calcifications et l'ossification. Ces lésions présentent une grande variation de localisation et des quantités de calcifications. Ce qui est pathognomonique pour la MOT à l'imagerie, est une périphérie bien circonscrite et fortement atténuée, souvent décrite comme plumeuse ou nuageuse, avec une partie centrale faiblement atténuée. Ces lésions peuvent être classifiées en stade précoce et stade tardif. Sur le CT scanner, le stade précoce présente peu de calcifications et des plans de clivage définitifs entre la lésion et l'os ou le muscle associé.

Le stade tardif se présente avec l'apparence pathognomonique décrite plus haut et la perte du plan de clivage suggestif de la fusion [6]. 
L'échographie peut être utile pour détecter une ossification focale lors du stade précoce.

La RMN révèle des caractéristiques dépendant du stade histologique. Au stade précoce, la lésion est iso-intense en T1 et hyper-intense en T2 [7]. L'analyse histologique des pièces opératoires lors de l'excision chirurgicale permet de confirmer le diagnostic de myosite ossifiante [8].

Le choix du traitement varie selon la littérature. Le seul traitement reconnu est l'excision chirurgicale complète de la lésion, suivi par de la physiothérapie intense et précoce. Le débat est de savoir quand réaliser la chirurgie. Beaucoup de chirurgiens recommandent un intervalle de 6 à 12 mois après l'identification de la lésion [9]. Cela permettrait d'exciser la lésion lors du stade mature, après formation complète des calcifications, et de diminuer ainsi le risque de récidive. Des cas de résolution spontanée ont aussi été rapportés. De plus, l'excision chirurgicale serait recommandée lorsque la lésion est accessible et en cas de gêne fonctionnelle [8]. Certains auteurs recommandent parfois d'éviter la chirurgie afin de prévenir un traumatisme additionnel dans la zone concernée [10].

Dans le cas présenté, il a été décidé d'attendre avant de proposer une intervention chirurgicale, étant donné que le seul symptôme apparent n'était pas une limitation de l'ouverture buccale, mais une douleur, sans doute due à l'arthrose condylienne liée au traumatisme antérieur.

La récidive de la lésion se produit dans $35 \%$ des cas. Celle-ci peut se produire lors d'une excision incomplète de la lésion [11].

Il existe également d'autres options de traitement pour prévenir une éventuelle récidive. L'étidronate, les anti-inflammatoires non-stéroïdiens, les stéroïdes, la warfarine et des radiations à faible dose ont été proposés [8, 11-13]. Les données de la littérature manquent afin de prouver l'efficacité de ces traitements. Il est essentiel de suivre ces patients afin de détecter précocement des signes d'inflammation, une limitation de l'ouverture buccale ou encore des signes de calcifications à la radiographie. 


\section{Conclusion}

La MOT des muscles de la face est une entité très rare. Le diagnostic est essentiellement basé sur l'histoire traumatique, l'examen clinique, l'imagerie et l'examen anatomo-pathologique. La radiographie panoramique est utile mais pas suffisante. Le CT scanner permet de localiser les calcifications et la formation d'un tissu osseux ectopique. Le CT scanner est également primordial pour le diagnostic différentiel. La RMN peut également être utile. Le traitement le plus efficace, à l'heure actuelle, est l'excision chirurgicale complète de la lésion, suivie d'une physiothérapie intense et précoce. Les données de la littérature manquent à l'heure actuelle pour prouver l'efficacité d'autres traitements. Beaucoup d'auteurs recommandent une période d'attente avant de prévoir une résection chirurgicale.

- $\quad$ Remerciements : aucun

- Source de financement: Cette étude n'a reçu aucun soutien financier

- Conflit d'intérêt: les auteurs ne déclarent pas de conflit d'intérêt.

- Approbation éthique: Ce rapport de cas n'a pas nécessité d'accord de comité d'éthique.

- Consentement éclairé: Ce rapport de cas n'a pas nécessité de consentement éclairé de la part du patient. Toutes les images sont anonymisées. Aucune donnée qui permettrait d'identifier le patient n'a été fournie.

\section{Contribution des auteurs}

\begin{tabular}{|l|l|}
\hline Auteur & Contributlon \\
\hline Braem D & $\begin{array}{l}\text { Documentation, investigation, rédaction, } \\
\text { correction. }\end{array}$ \\
\hline Darche V & Découverte du cas, documentation. \\
\hline Legros E & Supervision, relecture, correction. \\
\hline
\end{tabular}




\section{Références}

1. Mevio E, Rizzi L, Bernasconi G, Mysositis ossificans traumatica of the temporal muscle: a case report. Auris Nasus Larynx 2001;28:345-347.

2. Guarda-Nardini L, Piccotti F, Ferronati G, Manfredini D. Myositis ossificans traumatica of the temporalis muscle: a case report and diagnosis considerations. Oral Maxillofac Surg 2012;16:221-225.

3. Spinzia A, Moscato G, Broccardo E, Castelletti L, Maglitto F, Orabona GD, Piombino P. A rare isolated unilateral myositis ossificans traumatica of the lateral pterygoid muscle: a case report. J Med Case Rep 2014;8:230.

4. Fité-Trepat L, Martos-Fernández M, Alberola-Ferranti M, Romanini-Montecino C, Saez-Barba M, Bescos-Atin C. Myositis ossificans of the masseter muscle: A rare location. Report of a case and review of literature. J Clin Exp Dent 2016;8:e210213.

5. Becker OE Becker OE, Avelar RL, Rivero ER, De Oliveira RB, Meurer MI, Santos AM, Haas Júnior OL, Meurer E. Myositis ossificans of the temporalis muscle. Head Neck Pathol 2016;10:340-344.

6. Schiff MJ, Meara DJ. Myositis ossificans of the temporalis muscle: case report and review of the literature. J Oral Maxillofac Surg 2013;71:1893-1898.

7. Ramieri V, Bianca C, Arangio P, Cascone P. Myositis ossificans of the medial pterygoid muscle. J Craniofac Surg 2010;21:1202-1204.

8. Touil H, Belghith A, Boudokhane M, Bouzaiene M. La myosite ossifiante du muscle temporal. Rev Stomatol Chir Maxillofac Chir Orale 2015;116:173-175.

9. Nemoto H, Sumiya N, Ito Y, Kimura N, Akizuki A, Maruyama N. Myositis ossificans traumatica of the masticatory muscles. J Craniofac Surg 2012;23:e514516.

10. Uematsu Y, Nishibayashi H, Fujita K, Matsumoto H, Itakura T. Myositis ossificans of the temporal muscle as a primary scalp tumor. Case report. Neurol Med Chir (Tokyo) 2005;45:56-58.

11. Manzano D, Silván A, Saez J, Moreno JC. Myositis Ossificans of the temporalis muscle. Case report. Med Oral Patol Oral Cir Bucal 2007;12:E277-280. 
12. Jayade B, Adirajaiah S, Vadera H, Kundalaswamy G, Sattur AP, Kalkur C. Myositis ossificans in medial, lateral pterygoid, and contralateral temporalis muscles: a rare case report. Oral Surg Oral Med Oral Pathol Oral Radiol 2013;116:e261-266.

13. Reddy SP, Prakash AP, Keerthi M, Rao BJ. Myositis ossificans traumatica of temporalis and medial pterygoid muscle. J Oral Maxillofac Pathol 2014;18:271-275. 